\title{
An elementary approach to the hypergeometric shift operators of Opdam
}

\author{
G. J. Heckman \\ Katholieke Universiteit, Mathematisch Instituut, NL-6525 ED Nijmegen, The Netherlands
}

Oblatum 2-IV-1990

\section{§1. Introduction}

A large part of spherical function theory on a Riemannian symmetric space can be generalized, after restriction to a maximal split torus, to the case where the root multiplicities of the restricted root system are allowed to be arbitrary real or complex parameters [ $\mathrm{HO}, \mathrm{He} 1, \mathrm{O} 1, \mathrm{O} 2, \mathrm{O} 3, \mathrm{He} 2]$. We have called these more general functions hypergeometric functions associated with a root system, since the rank one case just amounts to the theory of the Gaussian hypergeometric function. Our approach was through differential equations, whose form was quite easy to conjecture in simple algebraic terms. However the existence of these differential equations only followed in the very end, and depended at several stages on transcendental arguments.

In this paper we give an elementary algebraic construction of these hypergeometric differential equations. We also find the associated shift operators as previously obtained by Opdam [O2]. The key tool is a global analogue of the Dunk1 differential-difference operators [Du, He 3]. Although these operators do not form a commuting family (as they do in the infinitesimal case) they turn out to be self adjoint. Once this fact is realized (and easily proved) the desired hypergeometric differential operators and their shift operators are constructed in a fairly straight forward way.

Recently Macdonald has constructed $q$-analogues of the Jacobi polynomials associated with a root system [Ma2]. These polynomials form a bridge between the spherical function theory for semisimple groups over a real and $p$-adic field. The $q$-analogue constant term conjectures, formulated by Macdonald several years ago, naturally fit into this frame work and even have appropriate generalizations $[\mathrm{Ma} 1,2]$. We hope that the results of this paper will also have natural $q$-analogues, which in turn might yield a solution to the Macdonald conjectures, analogously to the work of Opdam in the case $q=1[\mathrm{O} 3]$. 


\section{§2. The operators $D_{\xi}$ are self adjoint}

Let $E$ be a real vector space of finite dimension, endowed with a positive definite symmetric bilinear form $(\cdot, \cdot)$. For $\alpha \in E$ with $\alpha \neq 0$ we write

$$
\alpha^{v}=\frac{2 \alpha}{(\alpha, \alpha)} \in E
$$

for the covector of $\alpha$ and

$$
r_{\alpha}(\lambda)=\lambda-\left(\alpha^{\nu}, \lambda\right) \alpha
$$

for the orthogonal reflection in the hyperplane perpendicular to $\alpha$.

Definition 2.1. An integral root system $R$ in $E$ is a finite set of non zero vectors in $E$ with $\left(\beta, \alpha^{\nu}\right) \in \mathbb{Z} \forall \alpha, \beta \in R$ and $r_{\alpha}(\beta) \in R \forall \alpha, \beta \in R$.

Let $R \subset E$ be an integral root system. We write $W=W(R)$ for the group generated by the reflections $r_{\alpha}, \alpha \in R$. Let $P=\left\{\lambda \in E ;\left(\lambda, \alpha^{v}\right) \in \mathbb{Z} \forall \alpha \in R\right\}$ be the weight lattice of $R$. We write $\mathbb{R}[P]$ for the group algebra over $\mathbb{R}$ of the free abelian group $P$. For each $\lambda \in P$ let $e^{\lambda}$ denote the corresponding element of $\mathbb{R}[P]$, so that $e^{\lambda} \cdot e^{\mu}=e^{\lambda+\mu},\left(e^{\lambda}\right)^{-1}=e^{-\lambda}$ and $e^{0}=1$, the identity element of $\mathbb{R}[P]$. The $e^{\lambda}, \lambda \in P$ form an $\mathbb{R}$-basis of $\mathbb{R}[P]$. The Weyl group $W$ of $R$ acts on $P$ and hence also on $\mathbb{R}[P]: w\left(e^{\lambda}\right)=e^{w \lambda \lambda}$ for $w \in W, \lambda \in P$. It is easy to see that for $\alpha \in R$ the operator

$$
\Delta_{\alpha}=\frac{1+e^{-\alpha}}{1-e^{-\alpha}} \circ\left(1-r_{\alpha}\right): \mathbb{R}[P] \rightarrow \mathbb{R}[P]
$$

is a well defined endomorphism of $\mathbb{R}[P]$. Clearly $\Delta_{-\alpha}=-\Delta_{\alpha}$ and $w \Delta_{\alpha} w^{-1}=\Delta_{w \alpha}$ for $\alpha \in R, w \in W$.

For $\xi \in E$ the partial derivative

$$
\partial_{\xi}: \mathbb{R}[P] \rightarrow \mathbb{R}[P]
$$

is defined by $\partial_{\xi}\left(e^{\lambda}\right)=(\lambda, \xi) e^{\lambda}$. Clearly the map $\xi \mapsto \partial_{\xi}$ is linear, and $w \partial_{\xi} w^{-1}=\partial_{w \xi}$ for $\xi \in E, w \in W$.

Definition 2.2. Suppose for $\alpha \in R$ we have given $k_{\alpha} \in \mathbb{R}$ with $k_{w \alpha}=k_{\alpha} \forall \alpha \in R, \forall w \in W$. Suppose $R_{+} \subset R$ is a fixed set of positive roots. For $\xi \in E$ we write

$$
D_{\xi}=D_{\xi}(k)=\partial_{\xi}+\frac{1}{2} \sum_{\alpha \in R_{+}} k_{\alpha}(\alpha, \xi) \Delta_{\alpha}: \mathbb{R}[P] \rightarrow \mathbb{R}[P] .
$$

Clearly the map $\xi \mapsto D_{\xi}$ is linear, and $w D_{\xi} w^{-1}=D_{w \xi}$ for $\xi \in E, w \in W$ (note that $D_{\xi}$ is independent of the choice of $R_{+} \subset R$ ).

Remark 2.3. The operator (2.5) is the global analogue of the Dunkl differentialdifference operators [Du, He3]. However in the infinitesimal case the operators $D_{\xi}, \xi \in E$ commute, whereas in the global case

$$
\left[D_{\xi}, D_{\eta}\right]=-\frac{1}{4} \sum_{\alpha, \beta \in R_{+}} k_{\alpha} k_{\beta}\{(\alpha, \xi)(\beta, \eta)-(\alpha, \eta)(\beta, \xi)\} r_{\alpha} r_{\beta} .
$$

This formula can be derived along the same lines as in [Du, He3]. We skip the proof since we do not need this result. Operators of the form (2.3) appeared in the work of Demazure on Schubert varieties [De 1,2], and their infinitesimal analogues were introduced by Berstein, Gel'fand and Gel'fand [BGG]. 
Definition 2.4. A function $k=\left\{k_{\alpha} ; \alpha \in R\right\}$ as in Definition 2.2 is called a multiplicity function on $R$. We say that $k$ is a non-negative integral multiplicity function on $R$ if

$$
\delta_{k}^{\frac{1}{2}}:=\prod_{\alpha \in R_{+}}\left(e^{\frac{1}{2} \alpha}-e^{-\frac{1}{2} \alpha}\right)^{k_{\alpha}} \in \mathbb{R}[P] .
$$

Clearly the set of all non-negative integral multiplicity functions on $R$ is closed under addition. Moreover if $k_{\alpha} \in 2 \mathbb{Z}_{+} \forall \alpha \in R$ then $k$ is certainly a non-negative integral multiplicity function on $R$.

For $f=\sum f_{\lambda} e^{\lambda} \in \mathbb{R}[P]$ with $f_{\lambda} \in \mathbb{R}$ and $f_{\lambda} \neq 0$ for only finitely many $\lambda \in P$ we write

$$
\begin{aligned}
& \bar{f}=\sum f_{-\lambda} e^{\lambda} \\
& C T(f)=f_{0} .
\end{aligned}
$$

Here $C T$ denotes the constant term.

Definition 2.5. For $k$ a non-negative integral multiplicity function on $R$ we put

$$
(f, g)_{k}=C T\left(f \bar{g} \delta_{k}^{\frac{1}{2}} \bar{\delta}_{k}^{\frac{1}{2}}\right) \quad f, g \in \mathbb{R}[P] .
$$

Proposition 2.6. For $k$ a non-negative integral multiplicity function on $R$ the formula (2.10) defines a positive definite symmetric bilinear form on $\mathbb{R}[P]$.

Proof. Clearly the formula (2.10) defines a symmetric bilinear form on $\mathbb{R}[P]$. Clearly the standard bilinear form $(f, g)=C T(f \bar{g})$ on $\mathbb{R}[P]$ is positive definite. Hence the form (2.10) is positive definite since $\mathbb{R}[P]$ has no zero divisors. Q.E.D.

It is easy to see that the inner product (2.10) has a (real) analytic continuation for $k_{\alpha} \geqq 0, \forall \alpha \in R$. The following theorem is one of the crucial ingredients for the main result of this paper.

Theorem 2.7. For all $\xi \in E$ the operator $D_{\xi}: \mathbb{R}[P] \rightarrow \mathbb{R}[P]$ given by (2.5) is self adjoint with respect to the inner product (2.10) on $\mathbb{R}[P]$, i.e.

$$
\left(D_{\xi} f, g\right)_{k}=\left(f, D_{\xi} g\right)_{k} \quad \forall f, g \in \mathbb{R}[P] .
$$

Proof. Observe that for $(f, g)=C T(f \bar{g})$ we have $\left(\partial_{\xi} f, g\right)=\left(f, \partial_{\xi} g\right) \forall f, g \in \mathbb{R}[P]$. Indeed this follows from $C T\left(\partial_{\xi}(f \bar{g})\right)=0$ and the fact that $\partial_{\xi}$ is a derivation of $\mathbb{R}[P]$. Hence the adjoint $D_{\xi}^{*}$ of $D_{\xi}$ with respect to the inner product $(2.10)$ is given by

$$
D_{\xi}^{*}=\left(\delta_{k}^{\frac{1}{2}} \bar{\delta}_{k}^{\frac{1}{2}}\right)^{-1} \circ\left\{\partial_{\xi}+\frac{1}{2} \sum_{\alpha \in R_{+}} k_{\alpha}(\alpha, \xi)\left(1-r_{\alpha}\right) \circ \frac{1+e^{\alpha}}{1-e^{\alpha}}\right\} \circ\left(\delta_{k}^{\frac{1}{2}} \bar{\delta}_{k}^{\frac{1}{2}}\right) .
$$

First observe that

$$
\begin{aligned}
\frac{1}{2} \sum_{\alpha \in R_{+}} k_{\alpha}(\alpha, \xi)\left(1-r_{\alpha}\right) \circ \frac{1+e^{\alpha}}{1-e^{\alpha}} & =\frac{1}{2} \sum_{\alpha \in R_{+}} k_{\alpha}(\alpha, \xi) \frac{1+e^{-\alpha}}{1-e^{-\alpha}} \circ\left(-1-r_{\alpha}\right) \\
& =-\sum_{\alpha \in R_{+}} k_{\alpha}(\alpha, \xi) \frac{1+e^{-\alpha}}{1-e^{-\alpha}}+\frac{1}{2} \sum_{\alpha \in R_{+}} k_{\alpha}(\alpha, \xi) \Delta_{\alpha} .
\end{aligned}
$$

If we write $\mathbb{R}[P]^{W}$ for the space of $W$-invariants in $\mathbb{R}[P]$ then it is clear that

$$
\Delta_{\alpha} \circ f=f \circ \Delta_{\alpha} \quad \forall f \in \mathbb{R}[P]^{W}, \quad \forall \alpha \in R
$$

as endomorphisms of $\mathbb{R}[P]$. 
Since

$$
\delta_{k}^{\frac{1}{\delta}} \bar{\delta}_{k}^{\frac{1}{2}}=\prod_{\alpha \in R}\left(e^{\frac{1}{2} \alpha}-e^{-\frac{1}{2} \alpha}\right)^{k_{\alpha}} \in \mathbb{R}[P]^{W}
$$

and

$$
\begin{aligned}
\left(\delta_{k}^{\frac{1}{2}} \bar{\delta}_{k}^{\frac{1}{2}}\right)^{-1} \circ \partial_{\xi} \circ\left(\delta_{k}^{\frac{1}{2}} \bar{\delta}_{k}^{\frac{1}{2}}\right) & =\partial_{\xi}+\sum_{\alpha \in R} k_{\alpha}\left(\frac{1}{2} \alpha, \xi\right) \frac{e^{\frac{1}{2} \alpha}+e^{-\frac{1}{2} \alpha}}{e^{\frac{1}{2} \alpha}-e^{-\frac{1}{2} \alpha}} \\
& =\partial_{\xi}+\sum_{\alpha \in R_{+}} k_{\alpha}(\alpha, \xi) \frac{1+e^{-\alpha}}{1-e^{-\alpha}}
\end{aligned}
$$

we find $D_{\xi}^{*}=D_{\xi}$. Q.E.D.

Remark 2.8. If we write the bracket $[\cdot, \cdot]$ for the commutator of endomorphisms of $\mathbb{R}[P]$ then it is clear from (2.12) that for $f \in \mathbb{R}[P]^{W}, \xi \in E$

$$
\left[D_{\xi}, f\right]=\left[\partial_{\xi}, f\right]=\partial_{\xi}(f) \text {. }
$$

This will be used in the next section to prove that certain endomorphism of $\mathbb{R}[P]^{W}$ are in fact differential operators.

\section{§3. Applications}

With $R_{+} \subset R$ a fixed set of positive roots we write

$$
P_{+}=\left\{\lambda \in P ;\left(\lambda, \alpha^{v}\right) \geqq 0 \forall \alpha \in R_{+}\right\}
$$

for the dominant weights, and

$$
Q_{+}=\left\{\lambda \in Q ;(\lambda, \mu) \geqq 0 \forall \mu \in P_{+}\right\}
$$

for the dual octant in the root lattice $Q$ of $R$.

We define a partial ordering on $P$ by

$$
\lambda \geqq \mu \quad \text { if and only if } \lambda-\mu \in Q_{+} .
$$

Since each $W$-orbit in $P$ meets $P_{+}$in exactly one point, it follows that the monomial symmetric functions

$$
m(\lambda)=\sum_{\mu \in W \lambda} e^{\mu}
$$

form an $\mathbb{R}$-basis of $\mathbb{R}[P]^{W}$ as $\lambda$ varies over $P_{+}$.

Definition 3.1. The Jacobi polynomials $p(\lambda)=p(\lambda, k) \in \mathbb{R}[P]^{W}$ are defined by

and

$$
p(\lambda)=\sum_{\mu \in P+, \mu \leqq \lambda} c_{\lambda \mu} m(\mu), \quad c_{\lambda \lambda}=1
$$

$$
(p(\lambda), m(\mu))_{k}=0 \quad \forall \mu \in P_{+}, \quad \mu<\lambda .
$$

The existence of the Jacobi polynomials with the two properties (3.4) and (3.5) is clear since $p(\lambda)$ is equal to $m(\lambda)$ minus the orthogonal projection of $m(\lambda)$ onto span $\langle m(\mu) ; \mu<\lambda\rangle$. Clearly the Jacobi polynomials $p(\lambda), \lambda \in P_{+}$also form an $\mathbb{R}$-basis of $\mathbb{R}[P]^{W}$. 
In case $k_{\alpha}=0 \forall \alpha \in R$ the Jacobi polynomials specialize to the monomial symmetric functions, and in case $R$ is reduced and $k_{\alpha}=1 \forall \alpha \in R$ the Jacobi polynomials become the Weyl characters.

Definition 3.2. A linear operator $L: \mathbb{R}[P]^{W} \rightarrow \mathbb{R}[P]^{W}$ is called triangular if

$$
\operatorname{Lm}(\lambda)=\sum_{\mu \in R_{+}, \mu \leqq \lambda} a_{\lambda \mu} m(\mu) .
$$

Proposition 3.3. If $L: \mathbb{R}[P]^{W} \rightarrow \mathbb{R}[P]^{W}$ is triangular and self adjoint (with respect to the inner product $(2.10)$ ) then the $p(\lambda)$ are eigenfunctions of $L$.

Proof. Since $L$ is triangular we have using (3.5)

$$
\begin{aligned}
L p(\lambda) & =\sum_{\mu \in P_{+}, \mu \leqq \lambda} c_{\lambda \mu} L m(\mu) \\
& =\sum_{\mu, v \in P_{+}, v \leqq \mu \leqq \lambda} c_{\lambda \mu} a_{\mu v} m(v) \\
& =\sum_{v \in P_{+}, v \leqq \lambda} b_{\lambda v} m(v)
\end{aligned}
$$

with coefficients $b_{\lambda v}$ given by $b_{\lambda v}=\sum_{\mu \in P_{+}, v \leqq \mu \leqq \lambda} c_{\lambda \mu} a_{\mu v}$.

Using that $L$ is self adjoint we get

$$
\begin{aligned}
(L p(\lambda), m(\mu))_{k} & =(p(\lambda), L m(\mu))_{k} \\
& =\sum_{v \in P_{+}, v \leqq \mu} a_{\mu v}(p(\lambda), m(v))_{k}=0
\end{aligned}
$$

if $\mu<\lambda$. Hence $L p(\lambda)=a_{\lambda \lambda} p(\lambda)$ Q.E.D.

Corollary 3.4. All self adjoint triangular linear operators on $\mathbb{R}[P]^{w}$ are simultaneously diagonalized by the Jacobi polynomials $p(\lambda)$, and therefore commute with each other.

For $\lambda \in P_{+}$we write

$$
C(\lambda)=\{\mu \in P ; w \mu \leqq \lambda \forall w \in W\}
$$

for the integral convex hull of $W \lambda$.

Proposition 3.5. For $\lambda \in P_{+}$fixed the linear space

$$
\left\{f=\sum f_{\mu} e^{\mu} \in \mathbb{R}[P] ; f_{\mu}=0 \quad \text { unless } \quad \mu \in C(\lambda)\right\}
$$

is invariant under the operators $D_{\xi}, \xi \in E$.

Proof. This is clear since the space (3.8) is easily seen to be invariant under both $\partial_{\xi}, \xi \in E$ and $\Delta_{\alpha}, \alpha \in R$. Q.E.D.

Notation 3.6. For $\xi \in E$ and $d \in \mathbb{Z}_{+}$we write

$$
D_{\xi, d}=\sum_{\eta \in W} D_{\eta}^{d}: \mathbb{R}[P] \rightarrow \mathbb{R}[P] .
$$

Clearly $w D_{\xi, d} w^{-1}=D_{\xi, d} \forall w \in W, \xi \in E, d \in \mathbb{Z}_{+}$and we write

$$
\operatorname{Res}\left(D_{\xi, d}\right): \mathbb{R}[P]^{W} \rightarrow \mathbb{R}[P]^{W}
$$

for the restriction of $D_{\xi, d}$ to $\mathbb{R}[P]^{W}$. 
Theorem 3.7. The operators (3.10) are self adjoint triangular linear operators on $\mathbb{R}[P]^{W}$, and therefore commute with each other.

Proof. Using Theorem 2.7 it is clear that the operators (3.9) are self adjoint on $\mathbb{R}[P]$. In particular their restriction $(3.10)$ to $\mathbb{R}[P]^{W}$ is self adjoint on $\mathbb{R}[P]^{W}$. The fact that the operators (3.10) are triangular is clear from Proposition 3.5. The theorem now follows from Corollary 3.4. Q.E.D.

Remark 3.8. If $\lambda_{1}, \ldots, \lambda_{n}$ are the fundamental weights in $P_{+}$(say rank $(R)=\operatorname{dim}(E)=n)$, then it is well known that

$$
\mathbb{R}[P]^{w}=\mathbb{R}\left[z_{1}, \ldots, z_{n}\right]
$$

with $z_{j}=m\left(\lambda_{j}\right)$ the fundamental monomial symmetric functions. By Remark 2.8 it is clear that for $f \in \mathbb{R}[P]^{W}$

and therefore

$$
\operatorname{ad}(f)^{d}\left(\operatorname{Res}\left(D_{\xi, d}\right)=\operatorname{ad}(f)^{d}\left(\operatorname{Res}\left(\sum_{\eta \in W \xi^{\xi}} \partial_{\eta}^{d}\right)\right)\right.
$$

$$
\operatorname{Res}\left(D_{\xi, \mathrm{d}}\right) \in \mathbb{R}\left[z_{1}, \ldots, z_{n}, \frac{\partial}{\partial z_{1}}, \ldots, \frac{\partial}{\partial z_{n}}\right]
$$

is a differential operator of order $d$ in the Weyl algebra with leading symbol $\operatorname{Res}\left(\sum_{n \in W \xi} \partial_{\eta}^{d}\right)$ independent of $k$.

Writing

$$
\operatorname{Res}\left(D_{\xi, d}\right)(p(\lambda))=\gamma\left(\operatorname{Res}\left(D_{\xi, d}\right)\right)(\lambda+\varrho) p(\lambda)
$$

with

$$
\varrho=\varrho(k)=\frac{1}{2} \sum_{\alpha \in R_{+}} k_{\alpha} \alpha \in P_{+}
$$

it is clear that $\lambda \mapsto \gamma\left(\operatorname{Res}\left(D_{\xi, d}\right)\right)(\lambda)$ is the restriction to $P_{+}$of a polynomial on $E$ of degree $d$, whose homogeneous part of degree $d$ equals

$$
\sum_{\eta \in W \xi}(\eta, \lambda)^{d}
$$

Example 3.9. If $\xi_{1}, \ldots, \xi_{n}$ is an orthonormal basis for $E$ then by a straight forward calculation one finds that

$$
\begin{aligned}
\sum_{j=1}^{n} D_{\xi_{j}}^{2}=\sum_{j=1^{\prime}}^{n} \partial_{\xi_{j}}^{2} & +\sum_{\alpha \in R_{+}} k_{\alpha} \frac{1+e^{-\alpha}}{1-e^{-\alpha}} \partial_{\alpha}-\sum_{\alpha \in R_{+}} k_{\alpha} \frac{(\alpha, \alpha)}{e^{\alpha}-e^{-\alpha}} \Delta_{\alpha} \\
& +\frac{1}{4} \sum_{\alpha, \beta \in R_{+}} k_{\alpha} k_{\beta}(\alpha, \beta) \Delta_{\alpha} \Delta_{\beta}
\end{aligned}
$$

is independent of the choice of the basis $\xi_{1}, \ldots, \xi_{n}$. In particular $\sum_{1}^{n} D_{\xi_{j}}^{2}$ commutes
with the action of $W$ and hence

$$
\operatorname{Res}\left(\sum_{1}^{n} D_{\xi_{j}}^{2}\right)=\sum_{1}^{n} \partial_{\xi_{j}}^{2}+\sum_{\alpha \in R_{+}} k_{\alpha} \frac{1+e^{-\alpha}}{1-e^{-\alpha}} \partial_{\alpha}
$$


is a well defined self adjoint triangular operator on $\mathbb{R}[P]^{W}$. By Corollary 3.4 and Theorem 3.7 it commutes with the operators (3.10). Observe also that (3.14) is a second order differential operator (as it should be) with

$$
\gamma\left(\operatorname{Res}\left(\sum_{1}^{n} D_{\xi_{j}}^{2}\right)\right)(\lambda)=(\lambda, \lambda)-(\varrho, \varrho) .
$$

Proposition 3.10. If $\mathbb{R}[E]^{W}$ denotes the algebra of $W$-invariant polynomials on $E$ then we have

$$
\gamma\left(\operatorname{Res}\left(D_{\xi, d}\right)\right) \in \mathbb{R}[E]^{W} .
$$

Proof. This was proved in [HO, Proposition 2.9] by an elementary algebraic argument. Q.E.D.

Theorem 3.11. For each $p \in \mathbb{R}[E]^{W}$ there exists a differential operator $D_{p}$ $=D_{p}(k) \in \mathbb{R}\left[k, z_{1}, \ldots, z_{n}, \frac{\partial}{\partial z_{1}}, \ldots, \frac{\hat{\partial}}{\partial z_{n}}\right]$ whose action on the Jacobi polynomiais $p(\lambda, k)$ is given by

$$
D_{p}(k) p(\lambda, k)=p(\lambda+\varrho(k)) p(\lambda, k), \quad \lambda \in P_{+} .
$$

If we write $\mathbb{D}=\mathbb{D}(k)=\left\{D_{p}(k) ; p \in \mathbb{R}[E]^{W}\right\}$ then the generalized Harish-Chandra homomorphism

$$
\gamma=\gamma(k): \mathbb{D} \rightarrow \mathbb{R}[E]^{W}
$$

defined by $\gamma\left(D_{p}\right)=p$ is an isomorphism of $\mathbb{R}$-algebras.

Proof. Only the first statement needs a proof. But this is proved by a standard argument with induction on the degree using the fact that the polynomials (3.13) as $\xi$ ranges over $E$ and $d$ over $\mathbb{Z}_{+}$generate the algebra of invariants $\mathbb{R}[E]^{W}$. Q.E.D.

An immediate consequence of this theorem is the orthogonality of the Jacobi polynomials originally obtained by transcendental methods [He1].

Corollary 3.12. The Jacobi polynomials satisfy the orthogonality relations

$$
(p(\lambda, k), p(\mu, k))_{k}=0 \quad \forall \lambda, \mu \in P_{+}, \quad \lambda \neq \mu .
$$

Proof. Given $\lambda, \mu \in P_{+}$with $\lambda \neq \mu$ there exists by Theorem 3.11 an operator $D \in \mathbb{D}$ with $\gamma(D)(\lambda+\varrho) \neq \gamma(D)(\mu+\varrho)$. Since $D$ is self adjoint and $D p(v)$ $=\gamma(D)(v+\varrho) p(v) \forall v \in P_{+}$the result follows from elementary linear algebra. Q.E.D.

We now describe how the Opdam shift operators can be obtained in an elementary way. For $S \subset R$ a $W$-orbit with $2 S$ not contained in $R$ we will construct the corresponding raising operator. If we put $S_{+}=R_{+} \cap S$ then it is easily verified that

$$
\Delta_{S}=\prod_{\alpha \in S_{+}}\left(e^{\frac{1}{2} \alpha}-e^{-\frac{1}{2} \alpha}\right) \in \mathbb{R}[P] .
$$

The Weyl denominator $\Delta_{S}$ associated with $S$ transforms under $W$ according to a one dimensional character $\varepsilon_{S}$, and every $f \in \mathbb{R}[P]$ which transforms under $W$ according to $\varepsilon_{S}$ is divisible in $\mathbb{R}[P]$ by $\Delta_{S}$. 
Notation 3.13. Let $r$ be the cardinality of $S_{+}$. For $\xi \in E$ we write

$$
E_{S, \xi, r}=\sum_{w \in W} \varepsilon_{S}(w) D_{w \xi}^{r}: \mathbb{R}[P] \rightarrow \mathbb{R}[P]
$$

Clearly $w E_{S, \xi, r} w^{-1}=\varepsilon_{S}(w) E_{S, \xi, r} \forall w \in W, \forall \xi \in E$ and therefore

$$
G_{S, \xi}=\operatorname{Res}\left(\Delta_{S}^{-1} E_{S, \xi, r}\right): \mathbb{R}[P]^{W} \rightarrow \mathbb{R}[P]^{W}
$$

is a well defined endomorphism of $\mathbb{R}[P]^{W}$.

Remark 3.14. As before we see that

$$
G_{S, \xi}=G_{S, \xi}(k) \in \mathbb{R}\left[k, z_{1}, \ldots, z_{n}, \frac{\partial}{\partial z_{1}}, \ldots, \frac{\partial}{\partial z_{n}}\right]
$$

is a differential operator of order $\leqq r$ in the Weyl algebra with leading symbol of order $r$ equal to $\Delta_{S}^{-1} \operatorname{Rest}\left(\sum_{w \in W} \varepsilon_{S}(w) \partial_{w \xi}^{r}\right)$ and independent of $k$. Using this it is easy to see that $G_{S, \xi} \neq 0$ for $\xi \in E$ regular for $W(S)$.

Theorem 3.15. The operator $G_{S, \xi}$ given by (3.20) satisfies the shift relation

$$
G_{S, \xi}(k) D(k)=D\left(k+1_{s}\right) G_{S, \xi}(k) \quad \forall D(k) \in \mathbb{D}(k) .
$$

Here $1_{S}$ is the multiplicity function on $R$ defined by $\left(1_{S}\right)_{\alpha}=1 \forall \alpha \in S$ and $\left(1_{s}\right)_{\alpha}=0 \forall \alpha \in R \backslash S$.

Proof. For $\lambda, \mu \in P_{+}$we have

$$
\begin{aligned}
& \left(G_{S, \xi}(k) p(\lambda, k), p\left(\mu, k+1_{S}\right)\right)_{k+1_{s}} \\
& \quad=\left(E_{S, \xi, r}(k) p(\lambda, k), \Delta_{S} p\left(\mu, k+1_{S}\right)\right)_{k} \\
& \quad=\left(p(\lambda, k), E_{S, \xi, r}\left(\Delta_{S} p\left(\mu, k+1_{s}\right)\right)\right)_{k}=0
\end{aligned}
$$

if $\mu+\varrho\left(1_{s}\right)<\lambda$ or equivalently $\mu<\lambda-\varrho\left(1_{s}\right)$. We conclude that $G_{s, \xi}(k) p(\lambda, k)$ is a multiple of $p\left(\lambda-\varrho\left(1_{s}\right), k+1_{s}\right)$, from which relation (3.21) is easily obtained.

Q.E.D.

It remains to construct the shift operator corresponding to a $W$-orbit $S \subset R$ with $2 S \subset R$. As observed by Opdam in his thesis this operator can be obtained from the raising operator corresponding to $2 S$ [O4, Bijgevoegde Stelling 2].

Proposition 3.16. Suppose the root system $R$ of type $B C_{n}$ decomposes under the action of $W$ as $R=S_{1} \cup S_{2} \cup S_{3}$ with $S_{2}=2 S_{3}$ and write $k_{1}, k_{2}, k_{3}$ for the multiplicities of roots in $S_{1}, S_{2}, S_{3}$ respectively. With $\Delta_{S_{3}}$ given by (3.17) the operator

$$
G_{S_{3}}(k)=\Delta_{S_{3}}^{3-2 k_{2}-2 k_{3}} \circ G_{S_{2}}\left(k_{1}, k_{2}, 1-2 k_{2}-k_{3}\right) \circ \Delta_{S_{3}}^{-1+2 k_{2}+2 k_{3}}
$$

satisfies the shift relation

$$
G_{S_{3}}(k) D(k)=D\left(k_{1}, k_{2}+1, k_{3}-2\right) G_{S_{3}}(k) \quad \forall D(k) \in \mathbb{D}(k) .
$$

Remark 3.17. Using formal algebraic properties of adjoints the complete family of shift operators can be obtained as in [O1]. 
Remark 3.18. Combining the work of Opdam [O 1,3] with the simple results of this paper brings the solution of the constant term conjectures of Macdonald back to the elementary level which their formulation requires.

\section{§4. Final remarks}

We start by making some historical comments. In the case that $k_{\alpha}$ equals half the root multiplicity of the restricted root system of a Riemannian symmetric space $G / K$ Theorem 3.11 is a consequence of the Harish-Chandra homomorphism for $G / K$ and the theory of the radial part [Ha, Hel 1, 2]. The possibility of generalizing spherical function theory to the case of arbitrary positive root multiplicities amounts in the rank one case to the theory of the classical hypergeometric function $F(\alpha, \beta, \gamma ; z)$. The fact that higher rank spaces admit a similar generalization seems to have been observed for the first time by Koornwinder, who did explicit calculations for root systems of type $A_{2}$ and $B C_{2}[K]$. Subsequently particular cases were dealt with by several people : commuting differential operators for type $A_{n}$ [Se, D, Ma 3], and shift operators for type $B C_{2}[\mathrm{~K}, \mathrm{Spr}]$, type $A_{2}[\mathrm{~V}]$, type $A_{3}[\mathrm{~B}]$. A complete discussion of the rank two case was given by Opdam [O1]. All methods were rather computational. Finally Theorem 3.11 and Theorem 3.15 were obtained in full generality by Opdam [O2, He1].

The commuting family of differential operators, as described by Theorem 3.11 , is transformed by conjugation with the function (2.7) into a commuting family of differential operators of which the second order one becomes the Schrödinger operator of the generalized Calogero-Moser system [HO, Prop. 2.2]. From this perspective Theorem 3.11 can be reformulated as the quantum complete integrability of the generalized Calogero-Moser system. Now the classical complete integrability follows from a classical limit [O2, Section 4]. Originally the classical complete integrability was obtained by Moser for type $A_{n}$ by realizing the system as a Lax pair [Mo]. This method was generalized by Olshanetsky and Perelomov for the classical root systems $A_{n}, B C_{n}$ [OP 1]. For an explanation of this point of view of dynamical systems we refer to the survey papers [OP2, OP 3, R].

It is quite likely that large parts of the harmonic analysis of spherical functions can be generalized to the context of this paper (say for $k_{\alpha} \geqq 0 \forall \alpha \in R$ ). For the compact case this now being established the next results to look for are the generalizations of the Paley-Wiener theorem and the Plancherel formula in the non compact case. One of the main ingredients for this seems to be an "explicit" generalization of the Abel transformation of Harish-Chandra.

I believe that ultimately this work will lead to a simpler and more complete understanding of the harmonic analysis of spherical functions on Riemannian symmetric spaces.

\section{References:}

[B] Beerends, R.: On the Abel transformation and its inversion. Proefschrift Leiden, 1987

[BGA] Bernstein, I.N., Gel'fand, I.M., Gel'fand, S.I.: Schubert cells and the cohomology of $G / P$. Russ. Math. Surveys 28, 1-26 (1973)

[D] Debiard, A.: Polynômes de Tchébychev et de Jacobi dans un espace Euclidien de dimension p. C.R. Acad. Sci. Paris 296, 529-532 (1983) 
[De1] Demazure, M.: Désingularisation des variétés de Schubert généralisés. Ann. Sci. Ec. Norm. Supér 7, 53-88 (1974)

[De2] Demazure, M.: Une nouvelle formule des caractères. Bull. Soc. Math. 98, 163-172 (1974)

[Du] Dunkl, C.F.: Differential-difference operators associated to reflection groups. Trans. AMS 311, 167-183 (1989)

[Ha] Harish-Chandra: Spherical functions on a semisimple Lie group I, Am. J. Math. 80, 553613 (1958), or the Collected Works, Vol. 2, pp. 409-478

[HO] Heckman, G.J., Opdam, E.M.: Root systems and hypergeometric functions I. Comp. Math. 64, 329-352 (1987)

[He 1] Heckman, G.J.: Root systems and hypergeometric functions II. Comp. Math. 64, 353-373 (1987)

[He2] Heckman, G.J.: Hecke algebras and hypergeometric functions. Invent. Math. 100, 403-417 (1990)

[He3] Heckman, G.J.: A remark on the Dunkl differential-difference operators, Proceedings of the Bowdoin conference on Harmonic analysis on reductive groups 1989

[Hel1] Helgason, S.: Differential Geometry, Lie groups and Symmetric Spaces. Academic Press: New York 1978

[Hel2] Helgason, S.: Groups and Geometric Analysis. Academic Press: New York 1984

[K] Koornwinder, T.H.: Orthogonal polynomials in two variables which are eigenfunctions of two algebraically independent differential operators, I-IV, Indag. Math. 36, 48-66 and 358-381 (1974)

[Ma1] Macdonald, I.G.: Some conjectures for root systems. Siam J. Math. Anal. 13, 988-1007 (1982)

[Ma2] Macdonald, I.G.: Orthogonal polynomials associated to root systems. Oxford 1988 (Preprint)

[Ma3] Macdonald, I.G.: Commuting differential operators and zonal spherical functions, Algebraic Groups Utrecht 1986, LNM vol. 1271, pp. 189-200

[Mo] Moser, J.: Three integrable systems connected with isospectral deformation. Adv. Math. 16, 197-220 (1975)

[O1] Opdam, E.M.: Root systems and hypergeometric functions III. Comp. Math. 67, 21-49 (1988)

[O2] Opdam, E.M.: Root systems and hypergeometric functions IV. Comp. Math. 67, 191-209 (1988)

[O3] Opdam, E.M.: Some applications of hypergeometric shift operators. Invent. Math. 98, 1-18 (1989)

[O4] Opdam, E.M.: Generalized hypergeometric functions associated with root systems, Proefschrift Leiden 1988

[OP1] Olshanetsky, M.A., Perelomov, A.M.: Completely integrable systems connected with semisimple Lie algebras. Invent Math. 37, 93-108 (1976)

[OP2] Olshanetsky, M.A., Perelomov, A.M.: Classical integrable finite dimensional systems related to Lie algebras. Phys. Reps. 71 (1981), 313-400.

[OP3] Olshanetsky, M.A., Perelomov, A.M.: Quantum integrable systems related to Lie algebras, Phys. Reps. 94, 313-400 (1983)

[R] Ruijsenaars, S.N.M.: Finite-dimensional soliton systems. In: Kupershmidt, B. (ed.), Integrable and superintegrable systems. World Scientific Singapore 1990

[Se] Sekiguchi, J.: Zonal spherical functions on some symmetric spaces. Publ. RIMS Kyoto Univ. 12, 455-459 (1977)

[Sp] Sprinkhuizen-Kuyper, I.G.: Orthogonal polynomials in two variables. A further analysis of the polynomials orthogonal over a region bounded by two lines and a parabola. Siam J. Math. An. 7 (4), 501-518 (1976)

[V] Vretare, L.: Formulas for elementary spherical functions and generalized Jacobi polynomials. Siam J. Math. An. 15 (4), 805-833 (1984) 